\title{
Distribution of Low-Density Lipoprotein and High-Density Lipoprotein Subclasses in Patients With Sarcoidosis
}

\author{
Jelena Vekic, PhD; Aleksandra Zeljkovic, PhD; Zorana Jelic-Ivanovic, PhD; Vesna Spasojevic-Kalimanovska, PhD; \\ Slavica Spasic, PhD; Jelica Videnovic-Ivanov, PhD; Jasmina Ivanisevic, BSC; Violeta Vucinic-Mihailovic, PhD; \\ Tamara Gojkovic, BSc
}

- Context.-Systemic inflammatory diseases are associated with proatherogenic lipoprotein profile, but there is a lack of information regarding overall distributions of lipoprotein subclasses in sarcoidosis.

Objective.-To investigate whether patients with sarcoidosis have altered distributions of plasma low-density lipoprotein (LDL) and high-density lipoprotein (HDL) particles.

Design.-Seventy-seven patients with biopsy-proven sarcoidosis (29 with acute and 48 with chronic sarcoidosis) treated with corticosteroids and 77 age- and sex-matched controls were included in the study. Low-density lipoprotein and HDL subclasses were determined by gradient gel electrophoresis, while inflammatory markers and lipid parameters were measured by standard laboratory methods.

Results. - Compared to controls, patients had fewer LDL I subclasses $(P<.001)$, but more LDL II and III $(P<.001)$ subclasses. This pattern was evident in both acute and

A bnormalities of lipoprotein metabolism have been documented in various diseases associated with proliferation of monocyte-macrophage system..$^{1,2}$ However, sarcoidosis, being a systemic inflammatory disease, ${ }^{3}$ has been scarcely investigated in terms of possible dyslipidemia. Previous studies have documented a link between sarcoidosis and certain impairments of lipid status parameters, particularly in total and high-density lipoprotein cholesterol (HDL-C) concentrations., ${ }^{4,5}$ As plasma lipoproteins comprise a complex spectrum of subfractions of different size, density, and lipid composition, ${ }^{6}$ contemporary research is focused on establishing a significance for lipoprotein subclass assess-

Accepted for publication March 20, 2013.

From the Department of Medical Biochemistry, Faculty of Pharmacy, University of Belgrade, Belgrade, Serbia (Drs Vekic, Zeljkovic, Jelic-Ivanovic, Spasojevic-Kalimanovska, and Spasic and Mses Ivanisevic and Gojkovic); and the Institute for Pulmonary Diseases and Tuberculosis, Clinical Centre of Serbia, Medical Faculty, University of Belgrade, Belgrade, Serbia (Drs VidenovicIvanov and Vucinic-Mihailovic).

The authors have no relevant financial interest in the products or companies described in this article.

Reprints: Jelena Vekic, PhD, Department of Medical Biochemistry, Faculty of Pharmacy, University of Belgrade, Vojvode Stepe 450, PO Box 146, Belgrade, Serbia (e-mail: jelena.vekic@pharmacy.bg.ac.rs). chronic disease groups. Patients also had smaller HDL size $(P<.001)$ and higher proportions of HDL 2a $(P=.006)$ and 3a particles $(P=.004)$. Patients with chronic sarcoidosis had smaller LDL size than those with acute disease $(P=.02)$ and higher proportions of HDL 3a subclasses $(P=.04)$ than controls. In acute sarcoidosis, relative proportions of $\mathrm{LDL}$ and $\mathrm{HDL}$ particles were associated with levels of inflammatory markers, whereas in chronic disease an association with concentrations of serum lipid parameters was found.

Conclusions. - The obtained results demonstrate adverse lipoprotein subfraction profile in sarcoidosis with sustained alterations during disease course. Evaluation of LDL and HDL particles may be helpful in identifying patients with higher cardiovascular risk, at least for prolonged corticosteroid therapy due to chronic disease course.

(Arch Pathol Lab Med. 2013;137:1780-1787; doi: 10.5858/arpa.2012-0299-OA)

ment in evaluation of dyslipidemia and its clinical consequences. Patients with other forms of systemic inflammatory diseases have proatherogenic low-density lipoprotein (LDL) and high-density lipoprotein (HDL) particles. ${ }^{7,8}$ On the other hand, there is a lack of information regarding overall distributions of LDL and HDL subfractions in patients with sarcoidosis.

Chronic inflammatory disorders have been associated with higher cardiovascular disease (CVD) risk, ${ }^{9}$ but there is a lack of data regarding sarcoidosis. Nevertheless, Kizer et al ${ }^{10}$ previously suggested a possible link between fibrotic lung diseases and increased prevalence of CVD. Furthermore, Ardic et al ${ }^{11}$ recently reported alteration in elastic properties of the aorta, and data from Ivanisevic et $\mathrm{al}^{12}$ revealed elevated oxidative stress, diminished antioxidative protection, and altered lipid profile in patients with sarcoidosis. The latest data from a prospective study including various forms of immune-mediated disorders demonstrated that patients with sarcoidosis also have significantly increased risk of hospitalization due to coronary artery disease, similar to patients suffering from other systemic inflammatory diseases. ${ }^{13}$ Sarcoidosis has various manifestations and variable course. It can be a mild and self-limited disease, but it can also be persistent and progressive, with more than one-third of patients suffering from a chronic disease 
course. $^{3}$ So far, differences in lipid and lipoprotein profiles between patients with acute or chronic form of sarcoidosis have been insufficiently investigated. Another significant aspect of sarcoidosis is pharmacologic treatment, primarily based on the use of corticosteroids. Previous investigations ${ }^{14}$ have suggested that short-term, low-dose corticosteroid treatment could have beneficial effects on HDL-C concentrations in patients with sarcoidosis. On the other hand, prolonged corticosteroid administration is known to induce dyslipidemia. ${ }^{15}$ To the best of our knowledge, there are no data about the impact of corticosteroid therapy for sarcoidosis on lipoprotein particle distributions.

The aim of this study was to evaluate HDL and LDL particle characteristics in a group of patients with sarcoidosis who were receiving corticosteroid therapy. In addition, we have examined the relationship of LDL and HDL particle sizes and subclasses with other disease-related parameters, as well as with the course of the disease.

\section{METHODS \\ Study Participants}

The study group consisted of 77 patients (23 males and 54 females; mean age, $49.9 \pm 10.1$ years) with biopsy-positive sarcoidosis. The patients were recruited from the Institute for Pulmonary Diseases and Tuberculosis, Clinical Centre of Serbia in Belgrade. A diagnosis was established in accordance with published criteria. ${ }^{16}$ At the time of recruitment, all patients underwent determination of serum angiotensin-converting enzyme (ACE) and calcium (Ca) levels, while medical records were used to obtain data about the patients' age, sex, disease course, chest radiologic stage (0-IV), and treatment.

Twenty-nine patients had acute sarcoidosis, while 48 patients had chronic sarcoidosis. Regarding clinical presentation of the disease, 53 patients had pulmonary sarcoidosis (48 patients with chest radiologic stage II and 5 patients with chest radiologic stage III) and 24 patients had extrapulmonary sarcoidosis ( 9 neurologic, 5 eye, and 10 heart manifestations; 20 with chest radiologic stage 0 and 4 with chest radiologic stage I). All the patients were treated with corticosteroids and/or immunosuppressants to control disease activity. Accordingly, $77.9 \%$ of patients were receiving prednisolone and $22.1 \%$ received combined prednisolone and methotrexate. The patients were treated with a low dose of prednisolone (median: $10 \mathrm{mg}$; interquartile range: $5-10 \mathrm{mg}$ ) during $24.5 \pm 16.4$ months (median: 24 months; interquartile range: $12-39$ months) and none of them received any lipid-lowering medications. The control group consisted of 77 age- and sex-matched healthy subjects (23 males and 54 females; mean age, $47.8 \pm 8.4$ years) who attended regular annual medical check-ups at various public health centers in Belgrade and were free of known cardiac, renal, and hepatic diseases. None of the subjects in this group used any prescribed medication. Both patients and controls were Serbian Caucasians.

Informed consent was obtained from each subject enrolled in the study. The study was planned and executed by following the ethical guidelines of the Helsinki Declaration. According to institutional guidelines, the study protocol was approved by the local institutional review board.

\section{Biochemical Analyses}

Blood samples were collected into evacuated tubes containing EDTA and serum sample tubes after a 12-hour fasting period. Plasma and serum were separated by immediate centrifugation at $1500 \mathrm{~g}$ for 10 minutes at $4^{\circ} \mathrm{C}$. Aliquots of each sample were stored at $-80^{\circ} \mathrm{C}$. The samples were thawed immediately before analyses.

Serum glucose, urea, creatinine, total protein, C-reactive protein (CRP), ACE, and total Ca were measured by standard laboratory methods. Total cholesterol and triglyceride (TG) concentrations were assayed by using enzymatic methods. The concentration of HDL-C was measured by using the same enzymatic method after precipitation of the plasma with phosphotungstic acid in the presence of magnesium ions. The concentration of LDL-C was determined by using a direct homogeneous assay (Olympus Diagnostica $\mathrm{GmbH}$, Hamburg, Germany). All biochemical parameters were measured by using an ILab 600 analyzer (Instrumentation Laboratory, Lexington, Massachusetts). Hypertriglyceridemia for subjects was defined as TG of at least $150 \mathrm{mg} / \mathrm{dL}$, while the criterion for low HDL-cholesterolemia was a HDL-C concentration of $40 \mathrm{mg} /$ $\mathrm{dL}$ or less in men and $50 \mathrm{mg} / \mathrm{dL}$ or less in women. ${ }^{17}$

\section{Low-Density Lipoprotein and HDL Subclass Determination}

Plasma LDL and HDL particles were separated by using a method previously described by Rainwater et al. ${ }^{18} \mathrm{~A}$ detailed description of the procedure has been published elsewhere. ${ }^{19}$ In brief, electrophoresis was performed at $8^{\circ} \mathrm{C}$ in a Hoefer SE 600 Ruby electrophoresis unit (Amersham Pharmacia Biotech, Vienna, Austria) with Tris-boric acid-disodium EDTA buffer, pH 8.35 for 20 hours. Gels were calibrated by using the Pharmacia highmolecular weight protein standards, carboxylated polystyrene microsphere beads, and human plasma with 2 LDL subclasses. After completion of electrophoresis, the lateral portions of the gels containing protein standards and carboxylated polystyrene microsphere beads were cut off and stained with Coomassie brilliant blue G-250 and the remaining parts of the gels containing plasma samples were stained overnight for lipids with Sudan black. Gels were analysed by using Image Scanner (Amersham Pharmacia Biotech) with Image Quant software (version 5.2, 1999; Molecular Dynamics, Sunnyvale, California, USA). The migration distance for each absorbance peak was determined and the particle diameter corresponding to each peak was calculated from the calibration curve. The estimated diameter of the major peak in the LDL and HDL regions of each scan was referred to as the dominant particle diameter. The size for LDL of $25.5 \mathrm{~nm}$ or less and HDL of $8.8 \mathrm{~nm}$ or less were the criteria used for the definition of smaller, denser LDL and HDL particles (i.e., LDL B and HDL 3 phenotypes). ${ }^{19}$ The relative content of each LDL and HDL subclass was estimated by determining the areas under the peaks of densitometric scans of plasma samples stained with Sudan black. ${ }^{20,21}$

\section{Statistical Analysis}

Data are shown as mean \pm standard deviation or mean \pm standard error of mean for normally distributed continuous variables and as relative or absolute frequencies for categorical variables. Since the distributions of ACE, TG, and CRP were skewed, variables were presented as median and interquartile range and logarithmically transformed before analysis. Differences in continuous variables were analyzed by using the Student $t$ test and analysis of variance with the Tukey post hoc test for subgroup differences. A correction for multiple hypothesis testing was performed by Tukey post hoc test, since multiple comparisons were performed after completing and analyzing overall data. Analysis of categorical variables was performed by using $\chi^{2}$ tests for contingency tables. Univariate associations were evaluated by using Spearman correlation analysis. Multiple regression analysis (enter selection) was used to seek possible independent association between LDL and HDL sizes and subclasses and course of the disease. A model was built up with disease course as dependent variable ( 0 , acute; 1 , chronic sarcoidosis) and age, sex, duration of therapy, dominant LDL and HDL diameters, and relative proportions of each particular LDL and HDL subclasses as independent variables. All independent variables were entered as continuous, except sex (0, female; 1 , male).

\section{RESULTS}

Compared to controls, patients with sarcoidosis had significantly higher concentrations of glucose, urea, and creatinine (Table 1). Expectedly, serum CRP levels were significantly higher in patients. We found no differences in total cholesterol and LDL-C concentrations between the groups. However, the patients had higher concentrations of 


\begin{tabular}{|c|c|c|c|}
\hline Age, y & $49.9 \pm 10.1$ & $47.8 \pm 8.4$ & .24 \\
\hline $\mathrm{ACE}, \mathrm{U} / \mathrm{L}^{\mathrm{b}}$ & $44.0(23.0-52.5)$ & $\ldots$ & $\ldots$ \\
\hline $\mathrm{Ca}, \mathrm{mg} / \mathrm{dL}$ & $9.44 \pm 0.44$ & $\cdots$ & $\begin{array}{l}\cdots \\
\cdots\end{array}$ \\
\hline CRP, $\mathrm{mg} / \mathrm{dL}^{\mathrm{b}}$ & $0.18(0.06-0.68)$ & $0.11(0.05-0.25)$ & .045 \\
\hline Glucose, mg/dL & $94.05 \pm 19.46$ & $85.05 \pm 13.15$ & .001 \\
\hline Creatinine, $\mathrm{mg} / \mathrm{dL}$ & $0.89 \pm 0.16$ & $0.81 \pm 0.15$ & .02 \\
\hline $\mathrm{TC}, \mathrm{mg} / \mathrm{dL}$ & $232.82 \pm 65.64$ & $226.64 \pm 50.97$ & .53 \\
\hline $\mathrm{LDL}-\mathrm{C}, \mathrm{mg} / \mathrm{dL}$ & $151.74 \pm 65.64$ & $149.03 \pm 45.17$ & .81 \\
\hline $\mathrm{HDL}-\mathrm{C}, \mathrm{mg} / \mathrm{dL}$ & $50.58 \pm 15.06$ & $55.59 \pm 12.36$ & .04 \\
\hline Low HDL-C, \% & 46.6 & 27.3 & .02 \\
\hline $\mathrm{TG}, \mathrm{mg} / \mathrm{dL}^{\mathrm{b}}$ & $156.64(115.93-215.04)$ & $105.31(79.65-171.68)$ & .001 \\
\hline Hypertriglyceridemia, \% & 57.4 & 31.2 & .003 \\
\hline
\end{tabular}

Abbreviations: ACE, angiotensin-converting enzyme; Ca, calcium; CRP, C-reactive protein; HDL-C, high-density lipoprotein cholesterol; LDL-C, low-density lipoprotein cholesterol; TC, total cholesterol; TG, triglycerides.

a For conversion from conventional to SI units multiply ACE by 16.667 ; Ca by 0.25 ; CRP by 10 ; glucose by 0.0555 ; total protein by 10 ; urea by 0.357 ; creatinine by 88.4 ; TC, LDL-C, and HDL-C by 0.0259 ; and TG by 0.0113 .

${ }^{\mathrm{b}}$ Data are presented as median (interquartile range).

TG, but lower HDL-C levels than controls. Accordingly, hypertriglyceridemia and low HDL-C levels were more prevalent in the patient group.

Characteristics of LDL and HDL particles in both study groups are shown in Table 2. No significant differences were found in dominant LDL particle diameters and in frequencies of LDL B phenotype between patients with sarcoidosis and controls. However, patients had significantly higher proportion of LDL II and III, but lower proportion of LDL I subclasses. Difference in the proportions of the smallest LDL IV particles did not reach statistical significance $(P=$ .06). In addition, patients with sarcoidosis had significantly smaller HDL size and higher frequency of HDL 3 phenotype. Analysis of HDL subclass distribution revealed increased proportions of HDL 2a and 3a particles in this group.

We found no significant differences in LDL and HDL particle sizes and subclasses between patients with pulmonary and extrapulmonary sarcoidosis (data not shown). In further analysis we have taken into account the course of the disease (Table 3). Patients with chronic sarcoidosis had significantly lower HDL-C concentration and smaller LDL size than patients with acute sarcoidosis. Serum CRP concentration was significantly higher in the group with acute sarcoidosis. Daily dosage of prednisolone was similar for both patients with acute and chronic sarcoidosis and treatment duration was significantly higher in the chronic sarcoidosis group.

Distributions of LDL and HDL subclasses in acute and chronic sarcoidosis are presented in Figure 1. Both groups of patients had more LDL II and LDL III, but less LDL I particles than controls (Figure 1, A). On the other hand, patients with acute sarcoidosis had significantly higher proportions of HDL 2a, while patients with the chronic form of the disease had significantly higher proportions of HDL 3a subclasses than control subjects (Figure 1, B). Figure 2 shows typical electrophoretic patterns of LDL and HDL particles in patients with acute and chronic sarcoidosis and controls.

Next, we examined the relationship of LDL and HDL particle sizes and subclasses with other laboratory parameters with respect to the disease course. In a group of patients with acute sarcoidosis $(\mathrm{n}=29)$, relative proportions of LDL II $(r=0.76 ; P=.02)$ and HDL $3 c(r=0.62 ; P=.03)$ subclasses were in positive correlation, while the proportion of LDL I $(r=-0.60 ; P=.04)$ particles were in negative correlation, with ACE activity. Concentration of CRP was negatively associated with proportion of LDL I $(r=-0.78$; $P$ $=.02$ ) subclasses (Figure 3, A) and positively associated with proportion of HDL 2a $(r=0.78 ; P=.02)$ particles (Figure 3, B).

We found no significant correlations of ACE and CRP concentrations with any of the lipid subfractions in patients with chronic sarcoidosis $(n=48)$. In the same group, HDL$C$ concentration was in positive correlation with LDL particle size $(r=0.39 ; P=.04)$, LDL I $(r=0.44 ; P=.04)$, and HDL $2 \mathrm{~b}(r=0.39 ; P=.04)$ subclass proportions, but in negative correlation with proportion of HDL 3c particles $(r=$ $-0.44 ; P=.03$ ). Relative proportions of HDL $3 c$ were positively associated $(r=0.40 ; P=.01)$, while proportions of HDL $2 \mathrm{~b}$ subclasses were negatively $(r=-0.33 ; P=.04)$ associated, with TG concentrations. For all patients, TG concentration was in significant positive correlation with duration of prednisolone therapy $(r=0.48 ; P=.03)$.

Multiple regression analysis was used to assess possible independent associations of LDL and HDL particle sizes and subclasses with the course of sarcoidosis (Table 4). The following variables were included in the analysis: age, sex, duration of the therapy, dominant LDL and HDL diameters, and relative proportions of each LDL and HDL subclasses. The obtained results showed that, among all examined LDL and HDL characteristics, only higher proportions of HDL 3a and HDL $3 c$ subclasses were significantly and independently associated with chronic sarcoidosis.

\section{COMMENT}

In this study we have demonstrated that patients with sarcoidosis receiving corticosteroid therapy have impaired distributions of LDL and HDL subclasses. To our knowledge, this is the first study to demonstrate an adverse lipoprotein subfraction profile in sarcoidosis, with sustained alterations during the disease course. Previous studies on dyslipidemia in sarcoidosis have focused exclusively on serum lipid parameters without further analysis of lipopro- 


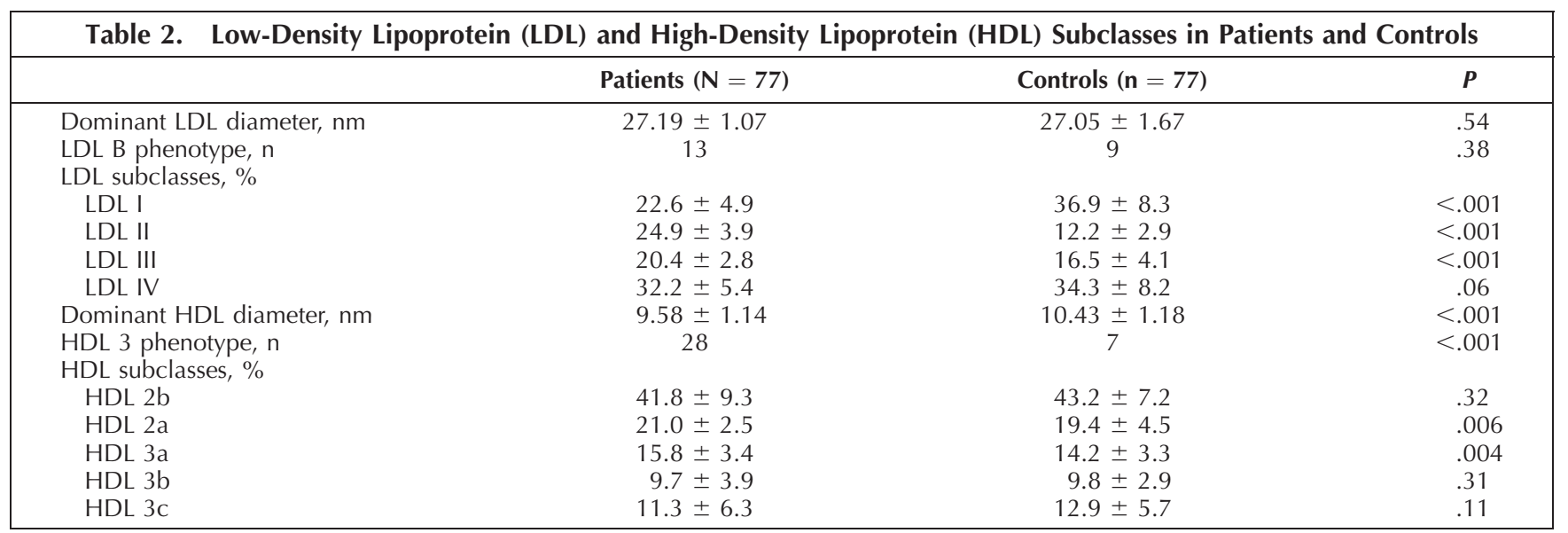

tein particle size heterogeneity. Kindman et $\mathrm{al}^{4}$ reported that patients with sarcoidosis have reduced levels of total cholesterol and HDL-C. Their findings were further confirmed in the study by Salazar et $a .^{5}$ In addition, the authors $^{5}$ also found that such decrease in HDL-C concentration mainly originates from the reduction of the cholesterol content in larger HDL 2 subfractions. Our current study extended earlier observations of Salazar et $\mathrm{al}^{5}$ by investigating overall distributions of LDL and HDL subclasses in this category of patients. Compared to controls, our patients with sarcoidosis had increased TG concentrations and reduced HDL-C levels, while total cholesterol and LDL-C levels did not differ between the 2 groups (Table 1). Lipoprotein subclass analysis revealed important changes in distinct LDL and HDL subspecies in the sarcoidosis group (Figure 2). The patients had reduced proportions of LDL I, but increased proportions of LDL II and small, dense LDL III particles (Table 2). This characteristic pattern was evident in both the acute and chronic sarcoidosis group (Figure 1, A). Similarly, there was a shift in HDL subclass distribution toward smaller particles in patients with sarcoidosis, when compared to controls (Figure 1, B). In the study of Salazar et $\mathrm{al}_{1}^{5}$ the relative proportion of HDL 3 particles, evaluated as HDL 3-C/HDL$\mathrm{C}$ ratio, was higher in patients with sarcoidosis than in controls, regardless of the disease activity. By resolving all HDL subclasses, we demonstrated that patients with sarcoidosis had increased proportions of HDL 3a and 2a particles (Table 2), providing further evidence for subtle disturbances of HDL metabolism in sarcoidosis, as already suggested by Salazar et al. ${ }^{5}$ The observed increase of smaller LDL and HDL particles in patients with sarcoidosis could be explained by hypertriglyceridemia, found in our patient group (Table 1). A possible reason could be that sarcoidosis per se is an inflammatory disease, and previous studies have demonstrated that the inflammatory state significantly influences metabolism of TG-rich particles, thus forcing a rearrangement of LDL and HDL subclass distributions toward smaller particles. ${ }^{22}$ On the other hand, all of our patients were treated with corticosteroids, which might further contribute to adverse alteration in lipoprotein distributions through induced insulin resistance and subsequent increased production of TG-rich particles. ${ }^{15}$

Although the presence of proatherogenic lipoprotein particles has been described in various systemic inflammatory diseases, ${ }^{7,8}$ this is the first study to report similar

\begin{tabular}{|c|c|c|c|}
\hline Age, y & $51.6 \pm 10.2$ & $49.0 \pm 10.1$ & .45 \\
\hline Dose of prednisolone, $\mathrm{mg}^{\mathrm{b}}$ & $5(5-10)$ & $10(5-10)$ & .43 \\
\hline Duration of therapy, $\mathrm{mo}^{\mathrm{b}}$ & $12(12-24)$ & $36(18-48)$ & .02 \\
\hline $\mathrm{ACE}, \mathrm{U} / \mathrm{L}^{\mathrm{b}}$ & $41.8(9.3-56.0)$ & $44.0(32.0-52.0)$ & .16 \\
\hline $\mathrm{Ca}, \mathrm{mg} / \mathrm{dL}$ & $9.48 \pm 0.48$ & $9.44 \pm 0.44$ & .75 \\
\hline CRP, $\mathrm{mg} / \mathrm{dL}^{\mathrm{b}}$ & $0.41(0.15-0.80)$ & $0.14(0.05-0.24)$ & .04 \\
\hline Glucose, mg/dL & $93.33 \pm 19.82$ & $94.59 \pm 19.64$ & .81 \\
\hline Total protein, $\mathrm{mg} / \mathrm{dL}$ & $7.13 \pm 1.49$ & $7.13 \pm 0.59$ & .99 \\
\hline Urea, mg/dL & $17.25 \pm 3.67$ & $16.05 \pm 5.21$ & .32 \\
\hline Creatinine, $\mathrm{mg} / \mathrm{dL}$ & $0.88 \pm 0.15$ & $0.90 \pm 0.16$ & .58 \\
\hline $\mathrm{TC}, \mathrm{mg} / \mathrm{dL}$ & $250.58 \pm 87.64$ & $221.24 \pm 44.40$ & .09 \\
\hline LDL-C, mg/dL & $169.88 \pm 96.91$ & $139.00 \pm 26.64$ & .16 \\
\hline $\mathrm{HDL}-\mathrm{C}, \mathrm{mg} / \mathrm{dL}$ & $58.69 \pm 16.99$ & $45.56 \pm 11.58$ & .006 \\
\hline $\mathrm{TG}, \mathrm{mg} / \mathrm{dL}^{\mathrm{b}}$ & $160.18(116.81-205.31)$ & $156.64(114.16-220.35)$ & .52 \\
\hline Dominant LDL diameter, nm & $27.55 \pm 0.99$ & $26.98 \pm 1.07$ & .02 \\
\hline Dominant HDL diameter, $\mathrm{nm}$ & $9.70 \pm 1.16$ & $9.51 \pm 1.14$ & .49 \\
\hline
\end{tabular}

Abbreviations: ACE, angiotensin-converting enzyme; Ca, calcium; CRP, C-reactive protein; HDL-C, high-density lipoprotein cholesterol; LDL-C, low-density lipoprotein cholesterol; TC, total cholesterol; TG, triglycerides.

a For conversion from conventional to SI units multiply ACE by 16.667 ; Ca by 0.25 ; CRP by 10 ; glucose by 0.0555 ; total protein by 10 ; urea by 0.357 ; creatinine by 88.4 ; TC, LDL-C, and HDL-C by 0.0259 ; and TC by 0.0113 .

${ }^{\mathrm{b}}$ Data are presented as median (interquartile range). 
Figure 1. Low-density lipoprotein ( $L D L)$ subclasses (A) and high-density lipoprotein $(H D L)$ subclasses $(B)$ in patients with acute and chronic sarcoidosis and control group. Data are shown as mean \pm standard error of mean. Presented $\mathrm{P}$ values indicate differences by overall analysis of variance. Subgroup differences were obtained by Tukey post hoc test: a, significantly different from control group $(\mathrm{P}<.001) ; b$, significantly different from control group ( $\mathrm{P}=.04)$; $\mathrm{C}$, significantly different from control group $(\mathrm{P}=.04)$.
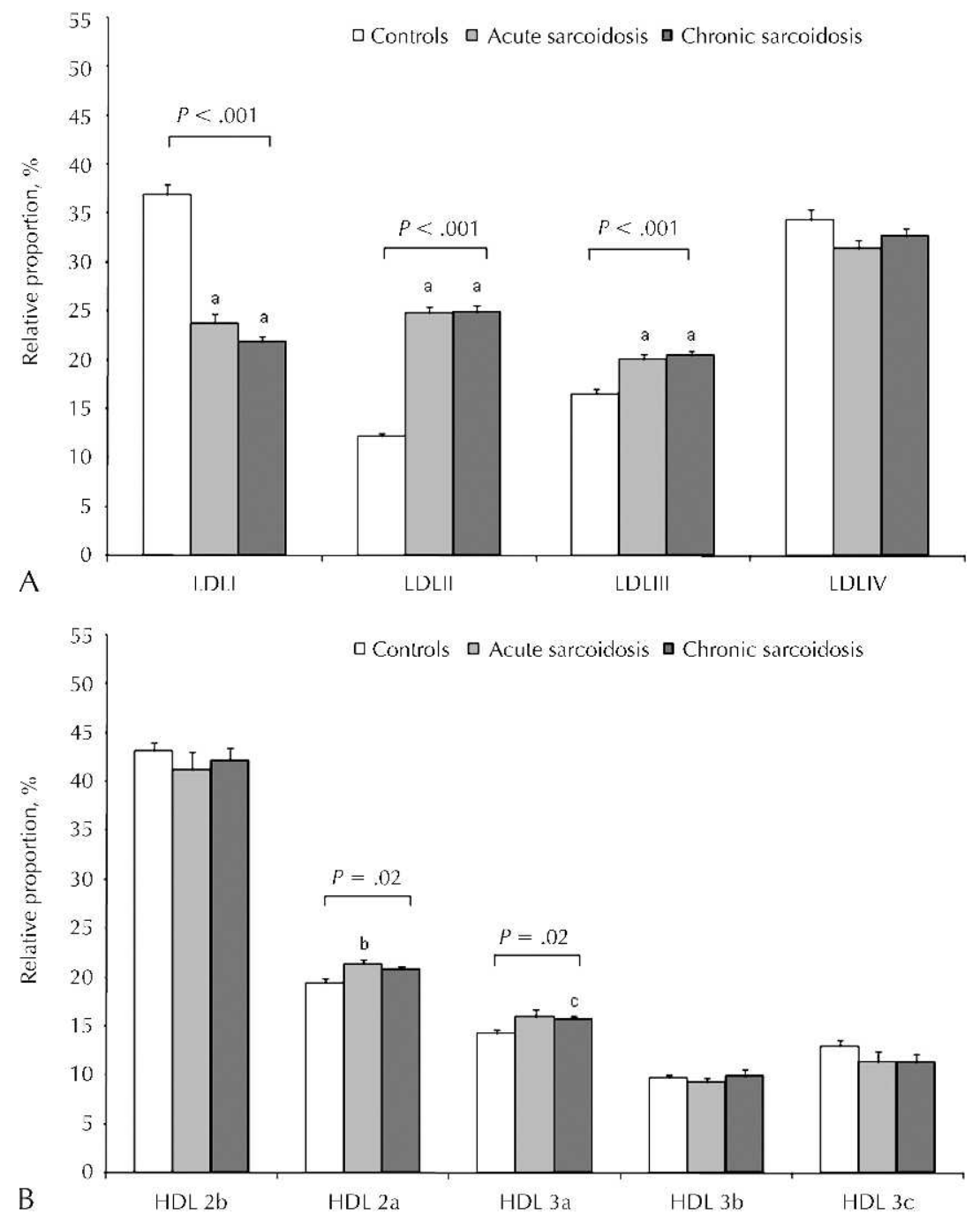

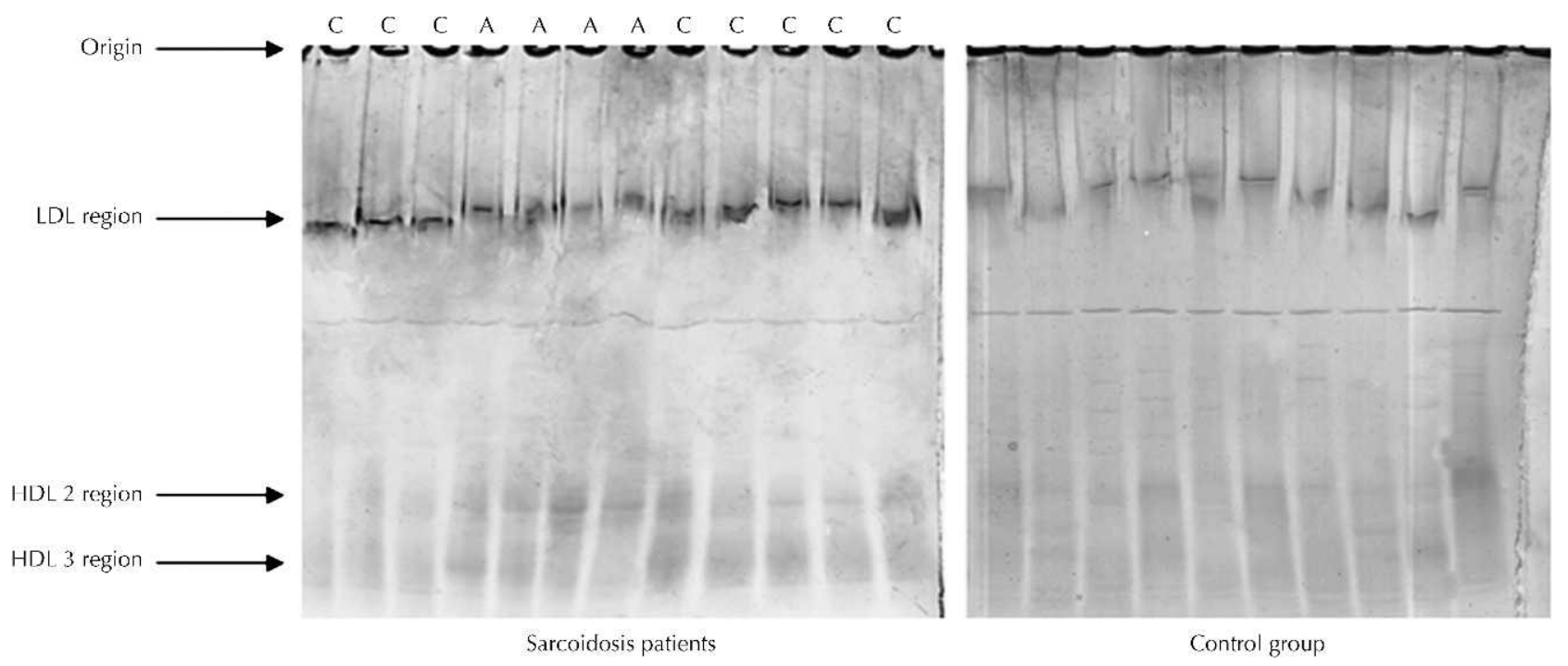

Figure 2. Electrophoretic patterns of low-density lipoprotein ( $L D L)$ and high-density lipoprotein (HDL) subclasses in patients with sarcoidosis and control group. A, Patient with acute sarcoidosis; C, Patient with chronic sarcoidosis. 


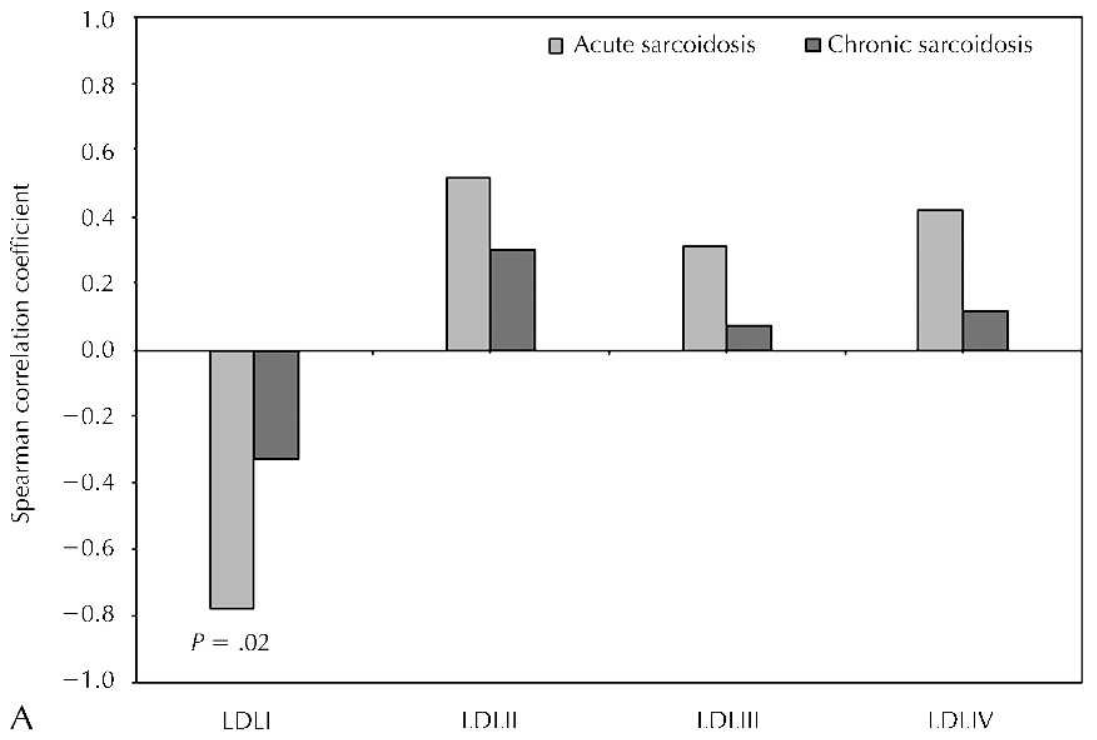

Figure 3. Correlations between C-reactive protein concentrations and relative proportions of lipoprotein subclasses in acute and chronic sarcoidosis. A, Low-density lipoprotein ( $L D L)$ subclasses. B, High-density lipoprotein (HDL) subclasses.

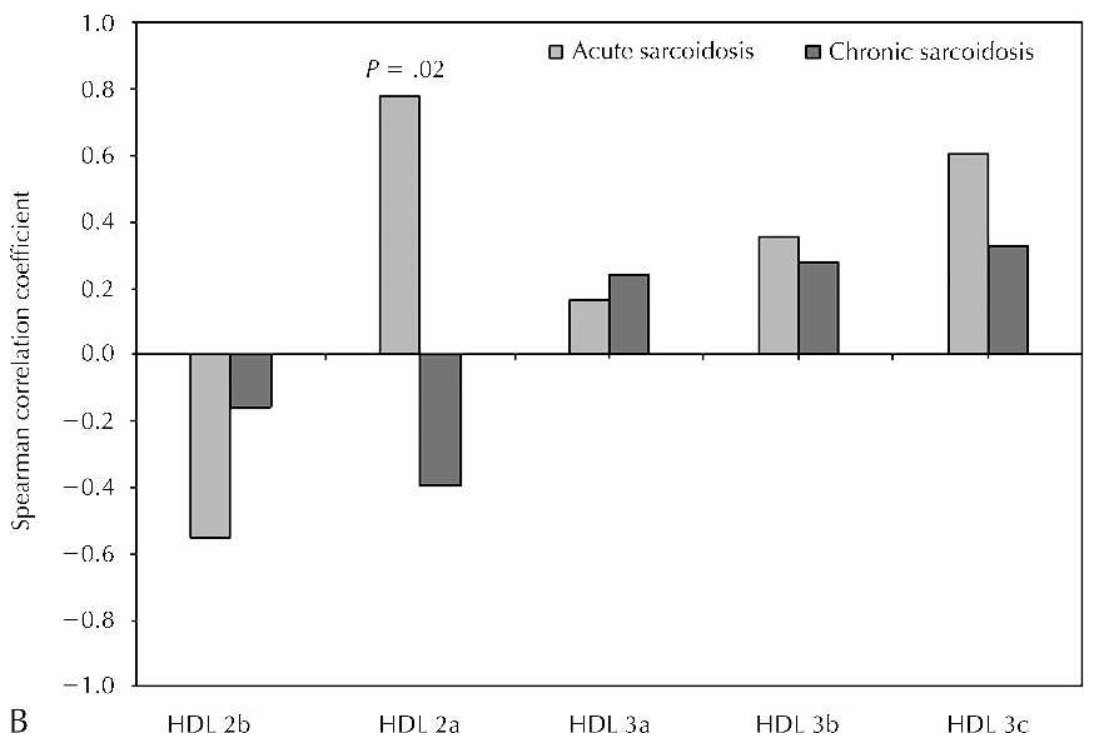

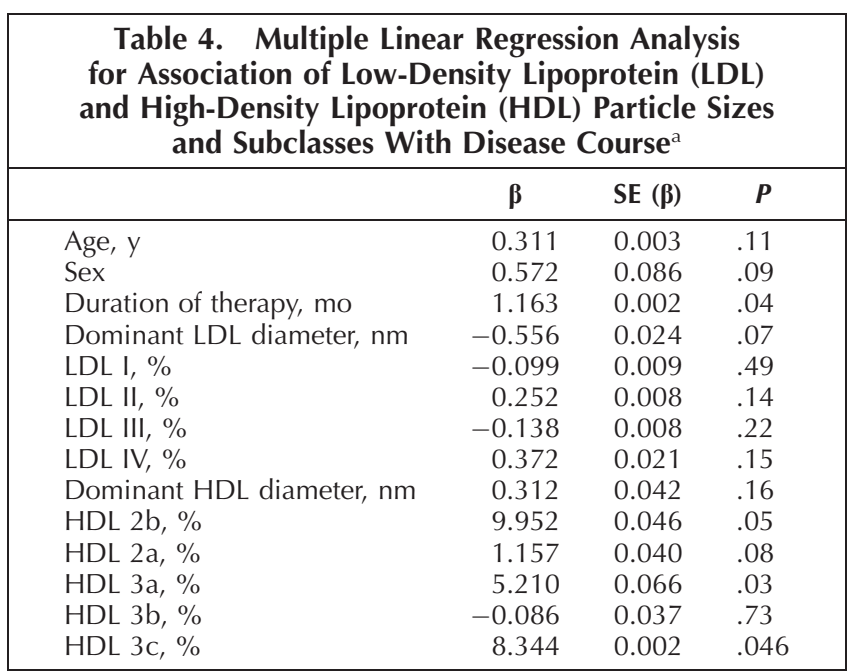

${ }^{a}$ Dependent variable was disease course $(0$, acute; 1 , chronic sarcoidosis). Independent variables were entered as continuous, except sex (0, female; 1 , male). changes in sarcoidosis. Alterations in LDL and HDL particle distributions, demonstrated in the current study (Table 2), correspond to the lipoprotein subclass profile regularly seen in other atherosclerosis-related diseases. ${ }^{8,20,21}$ Moreover, Van et $\mathrm{al}^{23}$ reported a similar proatherogenic lipoprotein phenotype in a considerable proportion of apparently healthy subjects. A cluster of lipoprotein abnormalities, including elevated TG, normal or slightly elevated LDL-C, reduced HDL-C concentrations, and preponderance of small, dense LDL and HDL particles, is generally referred to as atherogenic dyslipidemia. ${ }^{6}$ Such LDL and HDL particles could promote higher CVD risk by several mechanisms. Small, dense LDL particles easily penetrate the arterial intima, reside longer in subendothelium, and are more prone to oxidation, as compared to their larger counterparts. ${ }^{6}$ Also, smaller HDL particles, even if they are considered as essentially protective, could have decreased antiatherogenic capacity in dyslipidemia and inflammation, ${ }^{24}$ both regularly seen in sarcoidosis. ${ }^{25}$ Given that laboratory assessment of atherogenic dyslipidemia could help in identifying subjects with increased CVD risk, it 
seems reasonable to propose the same approach for patients with sarcoidosis.

Previous studies investigating lipid profiles in sarcoidosis focused exclusively on patients who did not receive corticosteroid therapy at the time they were evaluated. ${ }^{4,5}$ Although the symptoms that necessitate corticosteroid therapy remain controversial, systemic therapy is clearly indicated for cardiac, neurologic, and eye disease, as well as for progressive pulmonary sarcoidosis, ${ }^{16}$ and all of these indications were presented in our patient group. Our study was not primarily focused on estimating the direct impact of corticosteroids on lipoprotein distributions in sarcoidosis, but it is most likely that such therapy is also responsible, at least in part, for the observed alterations in LDL and HDL particle characteristics. Thus, our results suggest that benefit of corticosteroids on disease progression should be weighed against potential cardiovascular risk due to side effects of the therapy. Sustained glucocorticoid treatment is known to promote hypertension, dyslipidemia, and glucose intolerance. ${ }^{15}$ The impact of corticosteroids on the cardiovascular system has been well documented for patients undergoing transplants, ${ }^{26}$ as well as for patients with systemic lupus and rheumatoid arthritis. ${ }^{27,28}$ Souverein and colleagues ${ }^{29}$ recently investigated the risk for CVD and cerebrovascular disease development in a large cohort of patients treated with glucocorticoids. The authors ${ }^{29}$ reported that patients receiving oral corticosteroid therapy have a 25\% higher CVD risk than patients treated with nonsystemic glucocorticoids. Still, the incidence and severity of adverse cardiovascular events associated with the use of oral corticosteroids have not been documented in the context of sarcoidosis. Although steroidsparing approaches have moved to front-line therapy for many other immune-mediated inflammatory diseases, corticosteroids remain the treatment of choice for sarcoidosis. $^{30,31}$ Since prolonged systemic corticosteroid medication is able to enhance CVD risk, treatment decisions should also involve a trade-off between benefit and harm. Heterogeneity of clinical phenotypes in patients with sarcoidosis suggests that therapeutic approach should be individualized. ${ }^{16}$ For that reason, screening for the presence of small, dense LDL and HDL particles in this category of patients may be useful for identification of patients with higher CVD risk and may contribute in directing adequate preventive measures.

Further analysis was performed by taking into account the disease course. In a group of patients with acute sarcoidosis, correlation analysis revealed that alterations in LDL and HDL subclass distribution were strongly associated with the extent of inflammation. In this group we found a significant inverse correlation between proportion of large LDL I particles and CRP concentration (Figure 3, A). In addition, higher CRP levels were associated with increase of HDL 2a particles (Figure 3, B), suggesting an impact of inflammation in both LDL and HDL remodelling. Besides inflammation, formation of granulomas is one of the principal characteristics of sarcoidosis. Serum ACE activity reflects the burden of granulomas and is considered as an index of the extent and activity of the disease. ${ }^{32}$ Our analysis showed that ACE activity in acute sarcoidosis positively correlated with relative proportions of LDL II and small, dense HDL 3 c subclasses, and negatively with the proportion of LDL I particles. Salazar et $\mathrm{al}^{5}$ already reported significant association between higher ACE activity and low HDL-C concentration in patients with active sarcoidosis. As granuloma formation implicates selective accumulation of lymphocytes and macrophages with consequent release of cytokines, ${ }^{33}$ these proinflammatory changes might further contribute to disturbances of lipoprotein metabolism.

It must be emphasized that lipoprotein alterations found in our patients with chronic sarcoidosis were more prominent than those observed for acute disease course. Patients with chronic sarcoidosis had significantly smaller LDL size and lower HDL-C concentration than patients with acute disease (Table 3 ). In line with previous results, our patients with chronic sarcoidosis had higher proportions of HDL 3a subclasses as compared to controls (Figure 1, B), suggesting a gradual rearrangement toward smaller HDL particles as disease persists. Lack of associations between lipoprotein subclasses and markers of inflammation and disease activity in the chronic disease group could be a consequence of anti-inflammatory effects of prednisolone therapy. Although glucocorticoid treatment reduces CRP level, it is still unknown how such reduction of CRP correlates with other cardiovascular risk factors. ${ }^{15}$ Also, the relationship between serum CRP levels and lipid parameters was shown as inconsistent in large epidemiologic studies. ${ }^{34}$ However, correlation analysis in this group showed that increase of smaller LDL and HDL particles was associated with increase of TG and decrease of HDL-C levels. This indicates that dyslipidemia could be the major determinant of particle redistribution in chronic sarcoidosis. Again, these results could be attributed to side effects of prolonged prednisolone therapy. Namely, apart from beneficial effects on inflammation, corticosteroid treatment exhibits different short-term and long-term effects on serum lipids. ${ }^{15} \mathrm{Al}$ though daily dosage of prednisolone was similar for both patients with acute and chronic sarcoidosis, treatment duration was significantly higher in the chronic sarcoidosis group (Table 3). In an earlier study that evaluated influence of corticosteroid therapy on HDL in sarcoidosis, Salazar et $\mathrm{al}^{14}$ found that short-term application ( $6 \pm 3$ months) of an average $20 \mathrm{mg}$ of prednisolone can significantly raise HDL$\mathrm{C}$ concentration, mainly owing to increase of the largest HDL 2 subfractions. At this point, it is important to underline that our patients with chronic sarcoidosis were receiving a lower dosage of the same corticosteroid, but over much longer periods of time (Table 3 ). Since duration and cumulative dose are important determinants of corticosteroid-induced dyslipidemia, ${ }^{15}$ this could be a possible explanation for the observed discrepancy between the results. Still, small HDL $3 a$ and HDL 3c subclasses were independently associated with chronic sarcoidosis even after corticosteroid therapy was included as a confounder in multiple regression analysis (Table 4). This clearly indicates that association between chronic sarcoidosis and smaller HDL particles goes beyond the solely steroid effects.

Although the link between sarcoidosis and development of atherosclerosis is not established with certainty, several mechanisms were proposed to provide a biological basis for their associations. First of all, atherosclerosis and progressive sarcoidosis are fibroproliferative processes that implicate common trigger mechanisms in the development of both pathologic conditions. ${ }^{10}$ Another possibility is that systemic disorders in sarcoidosis, including chronic inflammation, dyslipidemia, and oxidative stress, could increase susceptibility for atherosclerosis development, as suggested by Ivanisevic et al. ${ }^{12}$ Taken together, a complex association between sarcoidosis and atherosclerosis should be further explored in larger, prospective studies to confirm risk factors 
and determine the true prevalence of CVD in this particular population.

Our study has some limitations. First, the findings presented were derived from patients of Serbian origin, which could limit generalization of our results and therefore should be extended in a larger sample with ethnically diverse patients. Second, owing to relatively small and nonuniform sample sizes in acute and chronic sarcoidosis subgroups, there is a possibility of missing associations that could otherwise be detected with larger study groups. Next, because all of the patients were receiving prednisolone therapy, our findings and conclusions cannot be extrapolated to untreated patients. Ideally, this study would have been better controlled with a group of subjects with some other inflammatory disorder treated with corticosteroids. However, it has been shown that patients with different forms of inflammatory diseases, even if untreated, have altered lipoprotein subclass distributions., ${ }^{7,8}$ The main objective of this study was to evaluate LDL and HDL particle characteristics in a group of patients with severe sarcoidosis that uniformly requires steroid therapy, which may account for the increased CVD risk. The reason for including the control group was to show that LDL and HDL particle distributions are worse in patients with sarcoidosis. Although our data do not permit us to conclude whether the observed differences are due to sarcoidosis itself, to treatment with corticosteroids, or both factors, presented findings point toward increased CVD risk in this category of patients, regardless of the cause. Finally, the cross-sectional nature of the study did not allow us to investigate a causal relationship between alterations of lipoprotein profile in sarcoidosis and development of atherosclerosis in later life. However, this study may offer some basic observations that could form the basis for future research.

In conclusion, the results presented here demonstrate altered LDL and HDL subclass distributions in patients with sarcoidosis. In addition, the current study is the first to describe sustained alterations in lipoprotein profile during disease course. Recognition and targeting of new markers associated with sarcoidosis could result in better understanding of the disease and improved patient care. In this manner, evaluation of LDL and HDL particle distribution may be helpful in identifying patients with higher cardiovascular risk, at least during prolonged corticosteroid therapy due to chronic disease course. Future studies should expand our preliminary observations in order to explore causality between chronic systemic inflammation, prescribed medications, and changes in lipid profile in sarcoidosis.

This work was supported by a grant from the Ministry of Education, Science and Technological Development, Republic of Serbia (Project No. 175035) and by the European Cooperation in Science and Technology (COST) BM0904 Action.

\section{References}

1. Ginsberg HN, Le NA, Gilbert HS. Altered high density lipoprotein metabolism in patients with myeloproliferative disorders and hypocholesterolemia. Metabolism. 1986;35(9):878-882.

2. Ginsberg HN, Goldberg I, Wang-Iverson P, et al. Increased catabolism of native and cyclohexanedione modified low density lipoprotein in subjects with myeloproliferative diseases. Arteriosclerosis. 1983;3(3):233-241.

3. Chen ES, Moller DR. Sarcoidosis: scientific progress and clinical challenges. Nat Rev Rheumatol. 2011;7(8):457-467.

4. Kindman LA, Gilbert HS, Almenoff JS, Ginsberg H, Fagerstrom R, Teirstein AS. High-density lipoprotein cholesterol is reduced in patients with sarcoidosis. Am / Med. 1989;86(4):376-378.
5. Salazar A, Maña J, Pinto X, et al. Low levels of high density lipoprotein cholesterol in patients with active sarcoidosis. Atherosclerosis. 1998;136(1):133137.

6. Rizzo M, Berneis K, Zeljkovic A, Vekic J. Should we routinely measure low-density and high-density lipoprotein subclasses? Clin Lab. 2009;55(11-12): 421-429.

7. Olusi SO, George S. Prevalence of LDL atherogenic phenotype in patients with systemic lupus erythematosus. Vasc Health Risk Manag. 2011;7:75-80.

8. Rizzo M, Spinas GA, Cesur M, Ozbalkan Z, Rini GB, Berneis K. Atherogenic lipoprotein phenotype and LDL size and subclasses in drug-naïve patients with early rheumatoid arthritis. Atherosclerosis. 2009;207(2):502-506.

9. Roifman I, Beck PL, Anderson TJ, Eisenberg MJ, Genest J. Chronic inflammatory diseases and cardiovascular risk: a systematic review. Can J Cardiol. 2011;27(2):174-182.

10. Kizer JR, Zisman DA, Blumenthal NP, et al. Association between pulmonary fibrosis and coronary artery disease. Arch Intern Med. 2004;164(5): 551-556.

11. Ardic I, Yarlioglues M, Dogdu O, et al. Assessment of aortic elastic properties in patients with sarcoidosis. Blood Press. 2012;21(5):286-292.

12. Ivanišević J, Kotur-Stevuljević J, Stefanović A, et al. Dyslipidemia and oxidative stress in sarcoidosis patients. Clin Biochem. 2012;45(9):677-682.

13. Zöller B, Li X, Sundquist J, Sundquist K. Risk of subsequent ischemic and hemorrhagic stroke in patients hospitalized for immune-mediated diseases: a nationwide follow-up study from Sweden. BMC Neurol. 2012;12:41-49.

14. Salazar A, Mañá J, Pintó X, Argimón JM, Hurtado I, Pujol R. Corticosteroid therapy increases HDL-cholesterol concentrations in patients with active sarcoidosis and hypoalphalipoproteinemia. Clin Chim Acta. 2002;320(1-2):5964.

15. Strohmayer EA, Krakoff LR. Glucocorticoids and cardiovascular risk factors. Endocrinol Metab Clin North Am. 2011;40(2):409-417.

16. The joint statement of the American Thoracic Society (ATS), the European Respiratory Society (ERS) and the World Association of Sarcoidosis and Other Granulomatous Disorders (WASOG). Statement on sarcoidosis. Am J Respir Crit Care Med. 1999;160(2):736-755.

17. Grundy SM, Cleeman II, Merz CN, et al. Implications of recent clinical trials for the National Cholesterol Education Program Adult Treatment Panel III guidelines. Circulation. 2004;110(6):227-239.

18. Rainwater DL, Moore $\mathrm{PH}$, Gamboa IO. Improved method for making nondenaturing composite gradient gels for the electrophoretic separation of lipoproteins. J Lipid Res. 2004;45(4):773-775.

19. Vekic J, Topic A, Zeljkovic A, Jelic-Ivanovic Z, Spasojevic-Kalimanovska V. $\mathrm{LDL}$ and $\mathrm{HDL}$ subclasses and their relationship with Framingham risk score in middle-aged Serbian population. Clin Biochem. 2007;40(5-6):310-316.

20. Zeljkovic A, Vekic J, Spasojevic-Kalimanovska V, et al. LDL and HDL subclasses in acute ischemic stroke: prediction of risk and short-term mortality. Atherosclerosis. 2010;210(2):548-554.

21. Zeljkovic A, Vekic J, Spasojevic-Kalimanovska V, et al. Characteristics of low-density and high-density lipoprotein subclasses in pediatric renal transplant recipients. Transpl Int. 2011;24(11):1094-1102.

22. Byrne CD. Triglyceride-rich lipoproteins: are links with atherosclerosis mediated by a procoagulant and proinflammatory phenotype? Atherosclerosis. 1999;145(1):1-15

23. Van J, Pan J, Charles MA, Krauss R, Wong N, Wu X. Atherogenic lipid phenotype in a general group of subjects. Arch Pathol Lab Med. 2007;131(11): 1679-1685

24. Tsompanidi EM, Brinkmeier MS, Fotiadou EH, Giakoumi SM, Kypreos KE. $\mathrm{HDL}$ biogenesis and functions: role of HDL quality and quantity in atherosclerosis. Atherosclerosis. 2010;208(1):3-9.

25. Salazar A, Maña J, Fiol C, et al. Influence of serum amyloid A on the decrease of high density lipoprotein-cholesterol in active sarcoidosis. Atherosclerosis. 2000;152(2):497-502.

26. Becker DM, Chamberlain B, Swank R, et al. Relationship between corticosteroid exposure and plasma lipid levels in heart transplant recipients. Am / Med. 1988;85(5):632-638.

27. Svenungsson E, Jensen-Urstad K, Heimburger M, et al. Risk factors for cardiovascular disease in systemic lupus erythematosus. Circulation. 2001; 104(16):1887-1893.

28. Wallberg-Jonsson S, Ohman ML, Dahlqvist SR. Cardiovascular morbidity and mortality in patients with seropositive rheumatoid arthritis in Northern Sweden. I Rheumatol. 1997;24(3):445-451.

29. Souverein PC, Berard A, Van Staa TP, et al. Use of oral glucocorticoids and risk of cardiovascular and cerebrovascular disease in a population based casecontrol study. Heart. 2004;90(8):859-865.

30. Baughman RP, Nunes $H$. Therapy for sarcoidosis: evidence-based recommendations. Expert Rev Clin Immunol. 2012;8(1):95-103.

31. Hamzeh NY, Wamboldt FS, Weinberger HD. Management of cardiac sarcoidosis in the United States: a Delphi study. Chest. 2012;141(1):154-162.

32. Gilbert S, Steinbrech DS, Landas SK, Hunninghake GW. Amounts of angiotensin-converting enzyme mRNA reflect the burden of granulomas in granulomatous lung disease. Am Rev Resp Dis. 1993;148(2):483-486.

33. Grunewald J, Eklund A. Role of CD4+ T cells in sarcoidosis. Proc Am Thorac Soc. 2007;4(5):461-464.

34. Albert MA, Glynn RJ, Ridker PM. Plasma concentration of C-reactive protein and the calculated Framingham coronary heart disease risk score. Circulation. 2003;108(2):161-165 